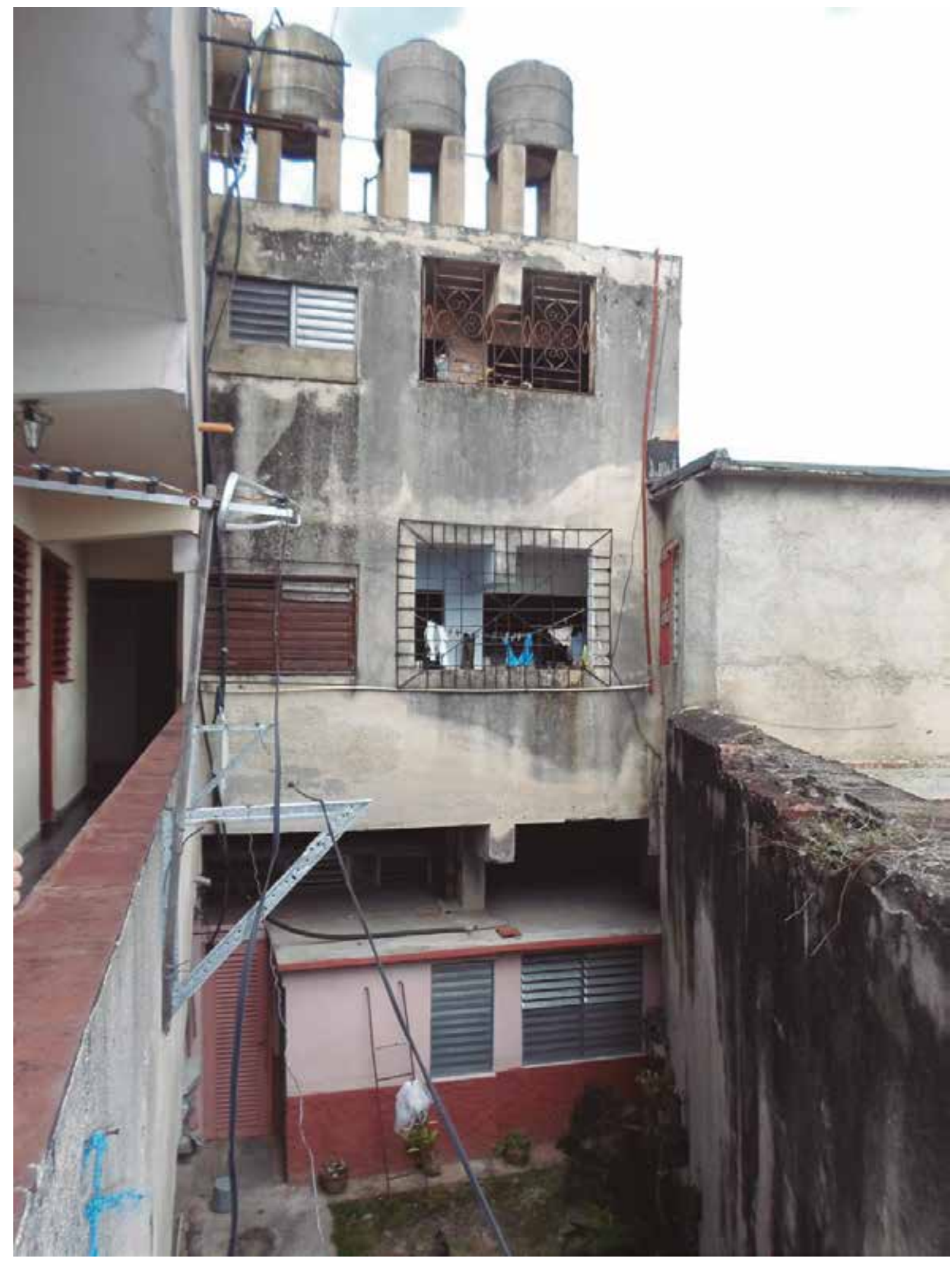

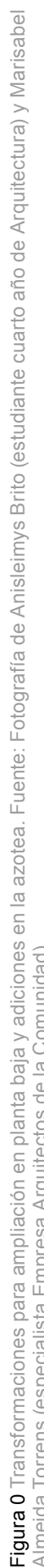




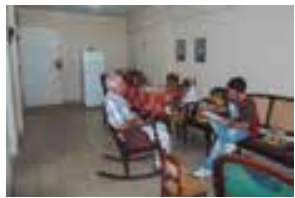

\title{
LOS EDIFICIOS DE APARTAMENTOS DEL MOVIMIENTO MODERNO EN CAMAGÜEY: UN VALIOSO PATRIMONIO A CONSERVAR DESDE LOS PROYECTOS Y CON LA PARTICIPACIÓN SOCIAL
}

\author{
MODERN MOVEMENT APARTMENT BUILDINGS IN CAMAGÜEY: PRESERVING \\ VALUABLE HERITAGE THROUGH PROJECTS AND SOCIAL PARTICIPATION ${ }^{1}$
}

Dianelis Falls Valdivieso ${ }^{2}$

RESUMEN

El centro histórico de Camagüey, declarado Patrimonio Cultural de la Humanidad en 2008, atesora un número considerable de edificios correspondientes al Movimiento Moderno, construidos en la década de 1950, que sufren actualmente modificaciones para satisfacer nuevas necesidades de la población que reside en ellos. Lamentablemente, las intervenciones en el patrimonio no siempre han contado con la asesoría técnica ni con la participación ciudadana necesarias para asegurar, por un lado, respuestas a los requerimientos de la población y, por otro, el resguardo del valioso patrimonio que atesora la ciudad. Ante esta problemática, los proyectos de investigación que articulan la participación de académicos-investigadores, profesionales y estudiantes, constituyen una vía eficaz para alcanzar soluciones acertadas. Mediante un análisis histórico-arquitectónico de los inmuebles, un diagnóstico detallado de las transformaciones y el criterio técnico de los profesionales, así como la incorporación de la población residente a través de las encuestas realizadas; se aborda aquí mecanismos para garantizar un pensamiento de respeto, (hacia los valores espaciales y ambientales que brindan las soluciones de estos inmuebles)valoración y sensibilización hacia las condiciones espaciales y ambientales que brindan las soluciones de estos inmuebles, las que están dirigidas a dar bienestar social a quienes los habitan y, al mismo tiempo, deben ser conservadas para legarlas a la sociedad.

Palabras clave: proyecto piloto, empresa estatal, desarrollo participativo, bienestar social, patrimonio.

\begin{abstract}
Camagüey's historic urban center, declared a World Heritage site in 2008, possesses a considerable number of Modern Movement buildings constructed in the decade of the 1950s, which have undergone modifications to fulfill the new needs of their residents. Regretfully, most of these transformations have not received the necessary technical advice or participation of citizens to ensure that both the population's requirements are met and the city's valuable heritage is protected. Faced with this problem, research projects that coordinate the participation of researcher-academics, professionals and students, are an effective way to achieve successful solutions. Through a historical-architectural property analysis, a detailed diagnostic assessment and the technical judgment of professionals, as well as the incorporation of the resident population via the surveys carried out, this paper addresses mechanisms to ensure an attitude of respect for, and appreciation and awareness of the spatial and environmental conditions that provide solutions for these properties, which are aimed at providing social welfare to those who live there and at the same time should be preserved to bequeath to society.
\end{abstract}

Keywords: pilot project, state-owned company, participatory development, social welfare, heritage.

[1] Artículo basado en resultados parciales del diagnóstico de los edificios de apartamentos del Movimiento Moderno del centro histórico de Camagüey. Tesis de Doctorado de la autora "Valores espaciales y ambientales de los edificios de apartamentos del Movimiento Moderno. Centro histórico de Camagüey", investigación que pertenece al Doctorado en Arquitectura de la CUJAE.

[2] Departamento de Arquitectura de la Universidad de Camagüey Ignacio Agramonte y Loynaz, Camagüey, Cuba. dianelis.falls@ reduc.edu.cu 


\section{INTRODUCCIÓN}

En la Universidad de Camagüey Ignacio Agramante Loynaz, se crea el Centro de Estudios de Conservación y Desarrollo de las Construcciones (CECODEC) que deriva de la fusión del Centro de Estudios de Conservación del Patrimonio CECONS y el Centro de Estudios de Estructuras Compuestas CENDES. Con dicha unión se amplía el campo de las investigaciones que se desarrollan y se atienden dos de las líneas priorizadas en la Universidad: Patrimonio y Vivienda y Construcción. La línea de investigaciones sobre patrimonio tiene bajo su responsabilidad el desarrollo de proyectos desde el nivel internacional hasta el empresarial y sus investigadores poseen más de 25 años de experiencia en el tema.

Así, el centro de estudio ha enfocado sus proyectos y otras acciones afines a la conservación del patrimonio cultural, enfrentándose, de una forma multilateral, interdisciplinaria e interinstitucional, a resolver problemas concretos de la práctica social y de las instituciones que solicitan determinadas investigaciones y que son beneficiarias de los resultados obtenidos. Específicamente, los estudios referidos al hábitat, como una de las prioridades del país para atender las crecientes necesidades de la población a través del mantenimiento, rehabilitación y otras categorías de intervención, vinculadas con la salvaguarda del patrimonio habitacional de los centros históricos, han sido especialmente considerados por la importancia que su solución reviste para las personas. 
Mediante el trabajo con la Empresa Arquitectos de la Comunidad se atiende a la protección de los inmuebles de valor patrimonial que se encuentran en el centro histórico de Camagüey, por ser esta entidad la encargada, en el caso cubano, de dar respuesta a las solicitudes de transformaciones realizadas por las personas residentes. En concreto, los edificios de apartamentos correspondientes al Movimiento Moderno están siendo intervenidos actualmente por la población que los habita $y$, en ocasiones, sin asesoramiento profesional ni el adecuado conocimiento de sus valores, lo que provoca serias modificaciones en su estructura espacial y volumétrica original. Por lo anterior, es necesaria, por un lado, la búsqueda de la documentación dispersa y fragmentada de estos inmuebles, como también el diagnóstico de las transformaciones ya realizadas a partir de las necesidades de la población y, por otro, la valoración de las características originales de los edificios, que deben conservarse manteniendo el equilibrio entre la adecuada respuesta a los requerimientos de los habitantes y la indispensable conservación de tan importantes exponentes del patrimonio.

\section{METODOLOGÍA}

Para llevar a cabo este proyecto, se utilizan diferentes métodos que ayudan a la recopilación de la información cuyo fin es detectar y evaluar los problemas a los que se les pretende dar solución. De los métodos empleados, resulta de vital importancia el analítico sintético, con un enfoque dialéctico, el que posibilita realizar un profundo análisis de las fuentes consultadas y arribar a la síntesis de los elementos esenciales a los efectos de la investigación. Desde una concepción holística y dialéctica, mediante el establecimiento de la relación observación-comprensión-interpretación, se alcanza, a partir del trabajo de campo, una primera aproximación a la problemática en estudio; lo que unido a la revisión de diferentes fuentes bibliográficas, a la información recopilada en los fondos de archivos, las entrevistas que se tienen con especialistas en el tema y las encuestas a la población, da origen a los resultados previstos en una primera etapa del diagnóstico.

En el estudio se trabaja con la totalidad de los edificios de apartamentos del Movimiento Moderno que fueron construidos en la década de 1950, los que constituyen 42 ejemplares y se encuentran en la trama urbana del centro histórico de la ciudad de Camagüey. Ellos conforman un universo considerable que por su centralidad, singularidad y valores debe ser salvaguardado. Se presenta, a modo de ejemplo, un caso de estudio, que evidencia las graves consecuencias que estos edificios sufren, al padecer las transformaciones realizadas por sus residentes sin el adecuado manejo de sus valores. Además, se emplea el método sistémico estructural que aborda la arquitectura como un todo sistémico determinando sus relaciones con el entorno y aislando del todo las variables necesarias (en la investigación la variable espacial y ambiental), así como sus relaciones entre sí y con otras variables, no menos importantes, como la técnico-constructiva y la expresión formal.

Participan en la labor dos investigadores del CECODEC, uno de la CUJAE, un profesional de la Empresa Arqui- tectos de la Comunidad, un grupo científico estudiantil curricular de segundo año de la carrera de Arquitectura (para el trabajo de campo que abarca el levantamiento fotográfico y la búsqueda de la documentación de archivo) y otro grupo científico estudiantil curricular de cuarto año de la misma carrera (para el diagnóstico de las transformaciones y la valoración crítica). Luego de seis meses es posible contar con la primera aproximación a la problemática, con lo cual la investigación alcanza resultados parciales en el diagnóstico y trabaja, hasta la fecha, en la caracterización tipológica de los inmuebles.

Como actividad de apoyo, se aplicaron encuestas a la población, que agruparon preguntas dirigidas fundamentalmente a identificar las características del núcleo familiar, el nivel de satisfacción con las condiciones del edificio y las nuevas necesidades que desean satisfacer e implican transformaciones al inmueble.

\section{RESULTADOS}

\section{El Movimiento Moderno en Cuba. Un acercamiento} a su surgimiento en el centro histórico de Camagüey

El Movimiento Moderno a nivel internacional tubo importantes aportes como lo fueron el empleo de los pilotes, la planta baja libre, el empleo de quiebrasoles, como elementos de protección solar, y las paredes ahuecadas, estas últimas muy usadas por autores latinoamericanos. Buscando la relación interior-exterior, fueron empleados en los diseños extensos paños de vidrio o curtainwall. Las ventanas miami fueron las más utilizadas, pues permitían el aprovechamiento de la iluminación y ventilación natural y, en menor grado, también se adoptó la ventana del tipo francesa.

El año 1947 marca el cambio más radical, al ocurrir el hecho simbólico de la quema del tratado de Viñola llevada a cabo por un grupo de estudiantes universitarios que no estaban de acuerdo con continuar estudiando la carrera de Arquitectura bajo los códigos academicistas. Sobre esta etapa, Segre planteó que "la disponibilidad de recursos existentes en este período se refleja en el quehacer constructivo promovido por la burguesía, que invierte la mayor parte de la plusvalía obtenida de las finanzas, el comercio y los latifundios, en apartamentos y viviendas individuales, cuya venta o alquiler produce grandes ganancias y una rápida amortización del capital" (1990:79).

En el caso cubano, Eugenio Batista fue uno de los profesionales que valoró altamente los elementos tradicionales coloniales y los reinterpretó junto a las nuevas tendencias, refiriéndose a ellos como las " $3 \mathrm{P}$ ", el patio, la persiana y los pórticos. Fueron estos elementos de vital importancia para asimilar las nuevas exigencias del Movimiento Moderno a las condiciones naturales y culturales de la nación y fueron empleados por los arquitectos tanto para el proyecto de obras de vivienda como para obras civiles. Las nuevas edificaciones que se proyectaron tomaron la vegetación como apoyo ambiental y en casos como el Cabaret Tropicana, el resulta- 
do fue realmente simbiótico. Respecto a la entrada en Cuba del Movimiento Moderno, Eduardo Luis Rodríguez plantea que, "por primera vez en su historia, la arquitectura cubana iguala y hasta aventaja en riqueza y calidad a la que se hacía paralelamente en otros países incluso desarrollados" (1997: 43).

Específicamente en la ciudad de Camagüey ocurre el florecimiento de esta nueva arquitectura, en la década de 1950, y fue el centro histórico la zona elegida para hacer alarde de las nuevas tecnologías y materializarlas en edificios. Algunos autores, como (Chaos, 2015: 658) postulan que "las zonas patrimoniales de una ciudad son las que concentran la esencia identitaria de un pueblo, una región o un país, pues perduran en sus espacios valores simbólicos, arquitectónicos y urbanos". Estas edificaciones se insertaron dentro de la trama urbana del centro histórico, y aunque, por un lado, se quiso respetar el perfil predominantemente bajo de esa zona (son notables los rasgos que destacan la horizontalidad), en determinados casos se observa una verticalidad en la primera planta, al emplear el mezanine o entresuelo como espacios para rentar o espacios para los negocios del dueño del edificio (Esquivel, 2011: 35), y otros en los que se incorporan determinados servicios como bancos, salones de belleza, comercios varios, entre algunas de las funciones que se pueden mencionar.

A pesar de que las soluciones formales que se dieron en la ciudad de Camagüey poseen características singulares, no son comparadas con las grandes torres vecinales de La Habana. Pero, aun así, es de reconocer que para la escala de la ciudad fueron construcciones que constituyeron importantes referentes de avances arquitectónicos.

\section{El proyecto empresarial y las investigaciones en el ámbito académico}

La Universidad de Camagüey Ignacio Agramante Loynaz ha obtenido importantes resultados en su trabajo con diferentes empresas en varios territorios del país. En específico, a través de los Centros de Estudios, ha contribuido a impulsar el desarrollo de investigaciones conjuntas con determinadas empresas y con la participación de la población residente en el centro histórico. Por solo citar algunas, pueden mencionarse la Oficina del Historiador de la Ciudad de Camagüey (OHCC), la Empresa Arquitectos de la Comunidad y la Delegación Provincial del MINTUR. Los resultados han sido reconocidos mediante la obtención de premios, publicaciones, participación en eventos y avales de introducción de resultados; ello ha servido para que empresas y organismos soliciten a la Universidad, concretamente a la Facultad de Construcciones su cooperación en dos proyectos empresariales con intereses en el centro histórico de la ciudad. Los proyectos de investigación se desarrollan a partir de necesidades reales y en busca de soluciones a las mismas. En este sentido, se incorporan académicos e investigadores de la Universidad, profesionales de las Empresas del territorio y estudiantes del pregrado y postgrado, alcanzándose muchos de los objetivos a través de tesis de pregrado, maestría y doctorado.

Asimismo, se ha desarrollado desde el 2014 el proyecto empresarial entre el MINTUR y el CECODEC, "Participación comunitaria en la gestión turística de destinos monumentales como vía para el desarrollo local", en el cual se trabaja con la comunidad que habita en los inmuebles de valor, para que ellos evalúen las afectaciones ambientales de sus lugares cotidianos de vida, especialmente, en edificios de apartamentos del Movimiento Moderno y en viviendas individuales de ejes importantes de la ciudad.

En el caso de la Empresa Arquitectos de la Comunidad se iniciaron acciones desde el año 2015, las que condujeron a la presentación de un proyecto empresarial en 2016, "Recomendaciones para mejorar progresivamente las condiciones de habitabilidad y mitigar las afectaciones a la imagen urbana de la ciudad de Camagüey"; espacio en el que se trabaja actualmente, ya con resultados parciales, en el diagnóstico de las transformaciones sufridas en los edificios de apartamentos del Movimiento Moderno del centro histórico de Camagüey, así como en el proceso de evaluación de sus valores espaciales y ambientales, teniéndose en cuenta el criterio de especialistas en el tema y de la población que habita en ellos.

Como parte de los proyectos están previstos resultados que corresponden a una tesis de pregrado, una de Maestría ${ }^{3}$ y dos de Doctorado ${ }^{4}$. Ambos proyectos constituyen un medio eficaz para garantizar, primero, la necesaria asesoría técnica que requiere la población para desarrollar acciones de trasformación en sus viviendas, sin afectar los valores de las mismas y, segundo, la fundamental articulación entre lo académico, lo empresarial (en este caso, las empresas que asumen la responsabilidad desde el gobierno) y lo comunitario; en busca de la satisfacción de la población, la preservación del patrimonio y la imagen cualificada de un valioso entorno considerado Patrimonio Mundial.

\section{La participación ciudadana, un componente indispensable para el diagnóstico}

Se coincide plenamente con Salgado cuando plantea que "el alojamiento del ser humano en condiciones básicas adecuadas es una de las principales tareas de la sociedad contemporánea" (2002: 22). Desde esa perspectiva, atender la problemática del hábitat, máxime cuando se habla de contextos de valor es una prioridad. Sin embargo, para dar soluciones a los disímiles factores que en ella inciden de manera desfavorable, no siem-

[3] La Tesis de Maestría en Conservación del Patrimonio "Caracterización de los edificios de apartamentos del Movimiento Moderno del centro histórico de Camagüey", responde al Proyecto Empresarial del MINTUR, y algunos de sus resultados (diagnóstico) se recogen en este artículo.

[4] Una de las Tesis de Doctorado, "Valores espaciales y ambientales de los edificios de apartamentos del Movimiento Moderno. Centro histórico de Camagüey", responde a las acciones conducentes al Proyecto Empresarial con la Empresa Arquitectos de la Comunidad y es una investigación que pertenece al Doctorado en Arquitectura de la CUJAE. 
pre se considera de vital importancia la participación ciudadana. Siguiendo a Caballero y Yordi (2009), es posible entender el proceso de desarrollo social como un proceso complejo que va más allá de solucionar solamente problemas sociales; se trata aquí de considerar la destrucción de espacios ya concebidos con una estructura diseñada para el confort social, ambiental, funcional y espiritual legada por el Movimiento Moderno, y también a las personas que buscan alternativas no siempre convencionales para dar respuesta a sus necesidades. Al analizar los edificios de apartamentos del Movimiento Moderno del centro histórico de la ciudad de Camagüey, se ha constatado que no han sido contempladas alternativas que garanticen una transformación del patrimonio respetuosa tanto de las actuales necesidades de la comunidad, como de la imagen de la ciudad tradicional -que se ve claramente amenazadade un centro histórico caracterizado por la presencia de obras notables dentro de su trama urbana.

Las modificaciones realizadas en inmuebles de valor y relevancia, dada su centralidad, se deben, entre otras causas, al crecimiento o la segregación del núcleo familiar que genera la subdivisión del inmueble original seguido de una agresiva intervención en planta y a nivel de fachada carente de criterios de integración. Ello provoca una pérdida de la expresión original y de muchos de los valores. Efectivamente, se reconoce en este escenario que "desde la percepción de los espacios, hasta el uso de los mismos responden a nuevos intereses y necesidades permeadas, muchas veces por el estilo de vida y el poder adquisitivo de los residentes en el inmueble. Se genera entonces el conflicto entre las necesidades crecientes de los residentes y la imagen urbana cada vez más transformada y deteriorada de la ciudad" (Mancebo, Chaos y Matamoros, 2015: 2).

Así, muchas veces se transforma el espacio creando otras áreas que al no haber estado concebidas desde un inicio van a ser desfavorables desde el punto de vista del confort creado por el propio Movimiento Moderno. Ejemplo de estas transformaciones pueden ser el crear habitaciones en patios de servicio ampliados o en patios interiores en plantas bajas, provocando que toda la ventilación natural diseñada para estos edificios se elimine, lo que lleva a que la población incluya ventilación mecánica, con los consiguientes aumentos en los costos de energía y la incorporación de aires acondicionados en las fachadas de los mismos, obviando quizás una mejor ubicación, nuevos muros, celosías, etc. y afectando considerablemente la imagen de la ciudad y la del propio edificio.

Según explica Rojas, cuando se selecciona un bien patrimonial "el mensaje es el punto inicial para determinar la individualidad y, más allá, la originalidad, para que la instalación tenga su propia identidad, con el fin de que sea recordada por quien la visita y se condicione deter- minado prestigio" (Rigol y Rojas, 2015: 540). A partir de este planteamiento, la participación ciudadana es de vital importancia en toda intervención para la salvaguarda de los valores patrimoniales, para identificar y reconocer los mismos y, en conjunto con la población residente y la asesoría técnica de especialistas, llegar a soluciones que resuelvan las necesidades sociales y no afecten los valores patrimoniales. Unido a ello, mediante la interacción se propicia el aprendizaje por parte de la población sobre dichos valores que ostentan los inmuebles y que los hacen dignos de ser conservados. Como plantea Antequera, "el patrimonio urbano que no se hace consciente en el imaginario, ni se encuentra protegido, es un patrimonio invisible, un patrimonio en riesgo" (2015: 9).

\section{Problemática social asociada a un caso de estudio. Edificio ubicado en la calle General Gómez $\mathrm{N}^{\circ} 3$ y 5 , entre Avellaneda y República, ciudad Camagüey}

El centro histórico de Camagüey atesora 42 inmuebles, entre los que se halla el edificio a trabajar, ubicado en la calle General Gómez № 3 y 5, entre Avellaneda y República, en el centro histórico de la ciudad de Camagüey. Este fue proyectado en el año 1956 y su ejecución se llevó a cabo en 1957. El inmueble se encuentra en la zona grado de protección II del centro histórico, presentando valor arquitectónico, carácter relevante $y$, desde luego, grado de protección II.

El inmueble presenta tres niveles: el segundo y tercero tienen cinco apartamentos cada uno y el primer nivel ${ }^{5}$, al ser creado el edificio, se reservó a un área de renta con tres espacios dispuestos para esta función. La fachada principal está ubicada al noreste, la parcela es de forma trapezoidal de $447.86 \mathrm{~m}^{2}$, de los cuales el $77,04 \%$ corresponde al área construida, que alcanza los $345,04 \mathrm{~m}^{2}$, y el espacio descubierto ocupa un área de $98,82 \mathrm{~m}^{2}$. Cada uno de los espacios en planta baja cuenta con baño; núcleos húmedos que están dispuestos de forma concentrada, incluyendo también el baño de la habitación del encargado. Miden alrededor de $2,85 \mathrm{~m}^{2}$ y la ventilación en dos de ellos es totalmente insuficiente, al ser ventilados mediante pequeñas ventanas por encima del baño del fondo, con el que comparten la medianería. Sin incluir estos baños, el primero de los espacios para rentar tiene $25 \mathrm{~m}^{2}$, el otro, $22,57 \mathrm{~m}^{2}$ y el tercero, $51,15 \mathrm{~m}^{2}$ aproximadamente. En la misma planta baja, pero en su parte posterior, el edificio cuenta además con cuatro espacios de parqueo en planta libre, lo cual se logra mediante el empleo de pilotes. Actualmente, los espacios de renta originales, se han convertido en viviendas y ello no siempre se ha llevado a cabo con las mejores soluciones de habitabilidad. El inmueble presenta un alto grado de deterioro que es visible tanto en el exterior como en el interior, lo que obviamente atenta contra el bienestar de sus habitantes. 


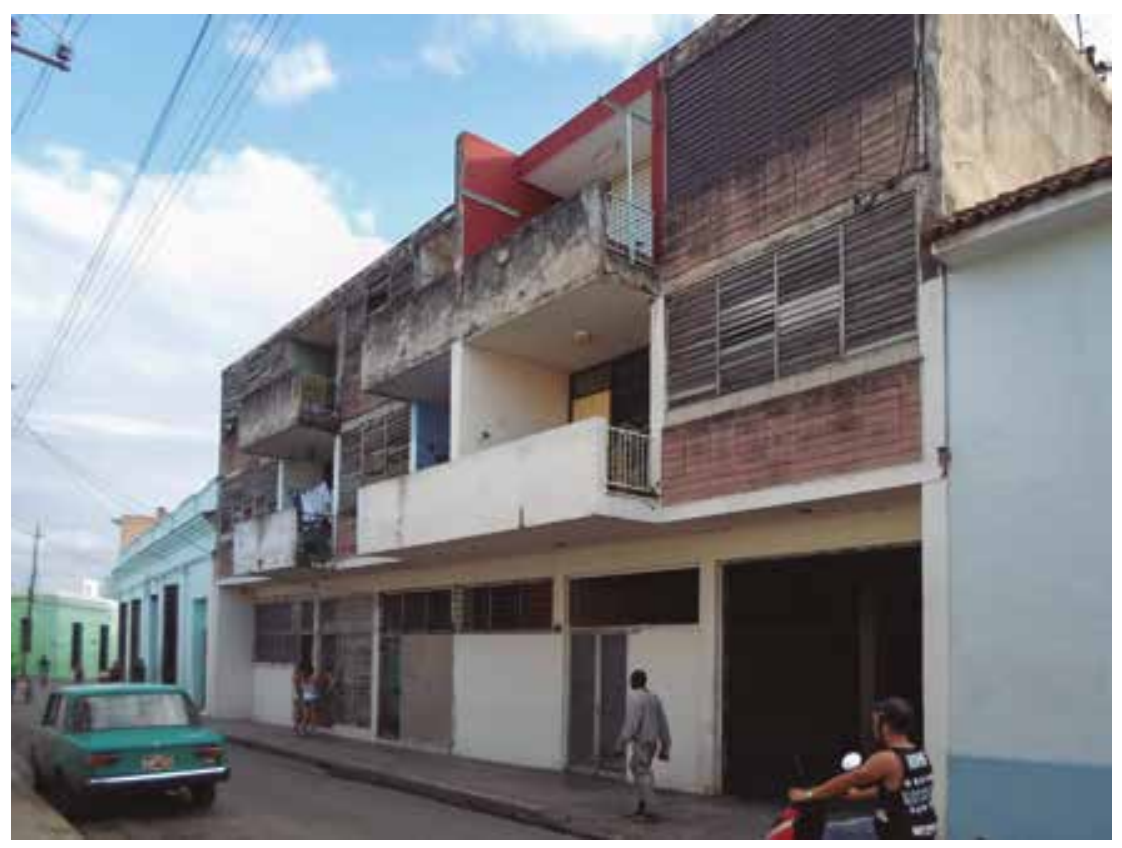

Figura 1 Exponente de la arquitectura del Movimiento Moderno en Camagüey. Fuente: Fotografía de la autora.
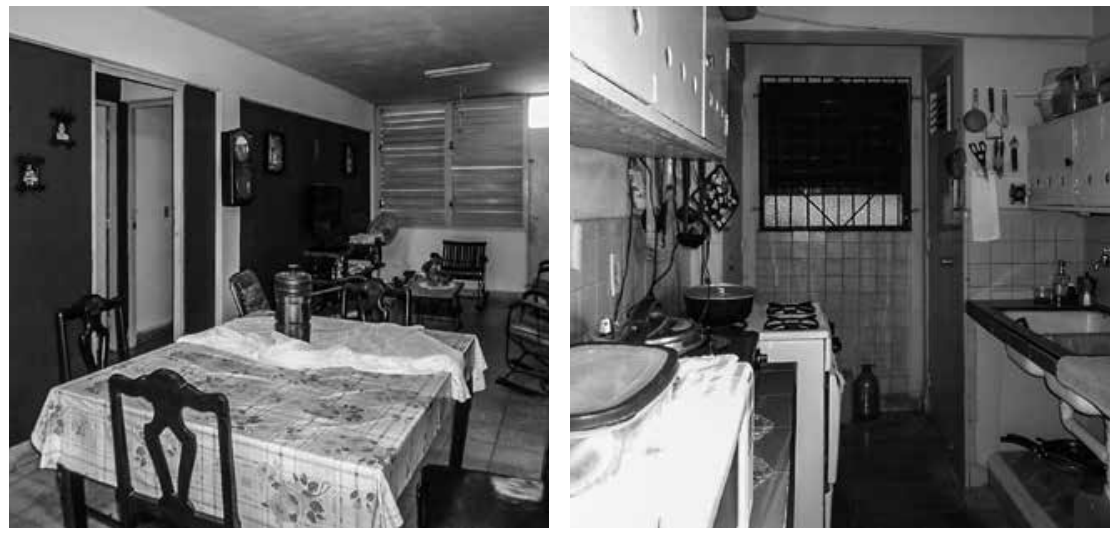

Figura 2 Interiores de uno de los apartamentos que conforman el edificio. Fuente: Fotografías de Anisleimys Brito y José Carlos Poleo (estudiantes cuarto año de Arquitectura). 
A la fachada principal, en el segundo y tercer nivel, dan tres apartamentos, que exhiben distintas intervenciones en los espacios interiores. Los apartamentos de los extremos presentan dos habitaciones, sala-comedor y terraza con una relación directa, baño intercalado entre las dos habitaciones, con acceso directo a un patio interior que brinda ventilación a la habitación posterior, $y$, al fondo, la cocina, directamente relacionada con el área de servicio que posee un medio baño. El apartamento del centro tiene una sola habitación con baño incluido y cuenta, también, con sala- comedor y terraza con vista al exterior, además de cocina y patio de servicio, los cuales se hallan conectados a un patio interior que posibilita la ventilación natural. Los apartamentos del frente disponen de dos accesos desde la calle, uno de servicio, por la cocina, y otro principal, por la sala comedor; ello evidencia la calidad espacial y ambiental.

El edificio muestra una expresión formal que rompe con el perfil urbano, que pese a tener puntales altos, presenta edificaciones circundantes son de un solo nivel; elemento este que caracterizó la entrada del Movimiento Moderno, de una manera "irreverente", en la ciudad tradicional. El diseño en la fachada se logra mediante una composición que emplea el cuadrado y el juego de llenos y vacíos (Figura 2) que armoniza con los enchapes de ladrillo a vista, propios de la arquitectura tradicional y que enmarcan las ventanas miami. Los balcones salen ligeramente de la fachada principal sin grandes alardes formales y expresivos. Su diseño es sencillo y aligerado, combinado con tramos de rejas, en busca de la simplicidad formal.

Los dos apartamentos ubicados al fondo son de una habitación, con baño incluido, y sus relaciones funcionales, en ambos casos directas, se establecen entre sala-comedor y cocina-patio-baño (Figura 3). Estos apartamentos solo tienen acceso desde la calle con entrada directa a la sala-comedor. Todos los espacios se encuentran ventilados mediante ventanas hacia el área de parqueo del edificio y a un patio interior.

La calidad espacial y ambiental se manifiesta a través de las soluciones que se logran. En todas las variantes se busca relacionar los espacios, atendiendo a su nivel de similitud e importancia; se relacionan directamente la sala-comedor con mayor fuerza y se proporciona una ubicación privilegiada en línea directa con la terraza lo que favorece la ventilación e iluminación natural. La cocina se relaciona con el comedor pero con menor grado de importancia dentro del espacio total del apartamento. El patio interior, propio de la vivienda tradicional, persiste como el encargado de canalizar todas las funciones circulatorias y garantizar la ventilación. Otro elemento positivo es que se tuvieron en cuenta soluciones para varios tipos de familias, diseñándose apartamentos de una y dos habitaciones.

\section{Participación comunitaria: resultados de las encuestas a la población}

Para el desarrollo de la investigación se trabajó con el total de los 13 apartamentos que conforman el edificio. Se seleccionó al $61,5 \%$ de sus residentes para aplicar la encuesta que se diseñó con el fin de identificar las características del núcleo familiar, el nivel de satisfacción de los habitantes con las condiciones del edificio y las nuevas necesidades que implican transformaciones en el inmueble, las que en muchas ocasiones, se han realizado sin la asesoría necesaria y son irreversibles (Figura 4).

Atendiendo a lo anterior, se identificó que el $65 \%$ de las personas encuestadas son universitarios. El $75 \%$ afirman que han vivido en el edificio más de 25 años. Las causas referidas para la permanencia en el inmueble están relacionadas con la ubicación que ocupa el edificio dentro del centro histórico, situación que les permite acceder a los servicios primarios, o la comodidad que representa que muchos de estos edificios cuentan con servicios diversos en el primer nivel. Las encuestas arrojaron que el motivo fundamental de las transformaciones es el crecimiento del núcleo familiar lo que se evidencia en el $50 \%$ de los encuestados. Las personas manifestaron satisfacción por su vivienda, debido a la distribución espacial, la presencia de balcones para la entrada de ventilación e iluminación natural. En este sentido, es factible entonces sostener la idea de la necesidad de educar para la conservación que "exige desarrollar en el individuo conocimientos, procedimientos y valores, de modo que garanticen un modo de actuación consecuente" (Guerra, Morales y De la Cruz, 2014: 32), con los valores del inmueble para que las soluciones a las necesidades de la población ocasionen las menores afectaciones al patrimonio. 

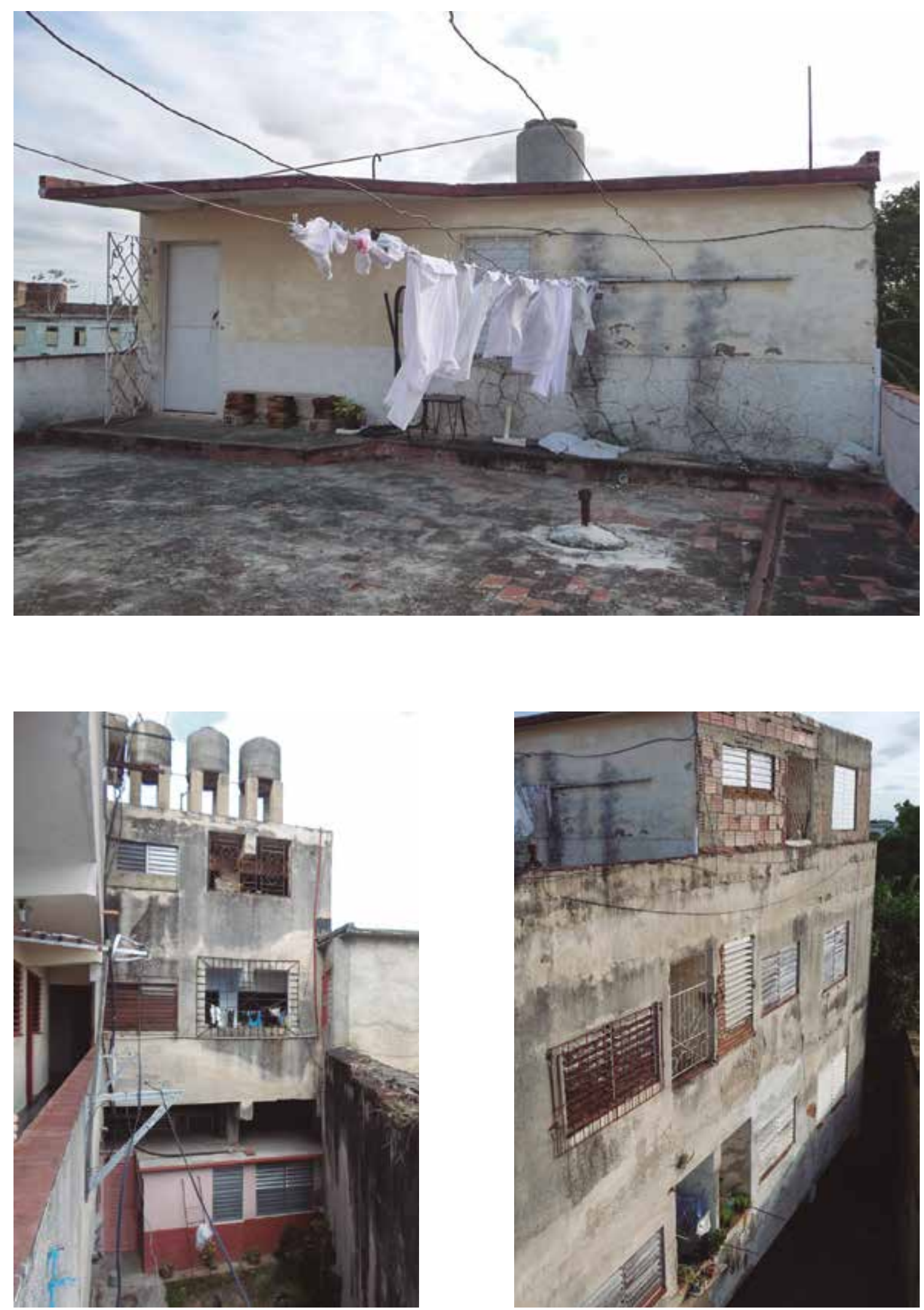

Figura 3 Transformaciones para ampliación en planta baja y adiciones en la azotea. Fuente: Fotografías de Anisleimys Brito (estudiante cuarto año de Arquitectura) y Marisabel Almeida Torrens (especialista Empresa Arquitectos de la Comunidad). 


\section{CONCLUSIONES}

La pérdida de los valores de los edificios diagnosticados, producto de transformaciones inadecuadas, no solo afecta la imagen de los mismos, sino también la calidad de vida de quienes los habitan, al perjudicar las condiciones ambientales que favorecen el confort del hábitat. El caso de estudio demuestra las implicaciones de dichas transformaciones $y$, como consecuencia, la necesidad de alcanzar soluciones que logren un equilibrio entre los actuales requerimientos de los residentes, que deben ser atendidos, y la de preservar los valiosos exponentes del movimiento moderno. El trabajo estima que las soluciones son posibles cuando se sensibiliza a la población respecto a los valores de sus espacios de vida y a la importancia de enfrentar las soluciones a sus necesidades con la incorporación del personal especializado para ello, lo cual se traduce en beneficio mutuo: de la sociedad por el legado que perdura y recibe, y de los residentes, al obtener la solución más adecuada a sus demandas. De allí la significación de los Proyectos Empresariales que permiten articular la presencia de académicos-investigadores, profesionales implicados, estudiantes y la población en la toma de decisiones sobre el bien patrimonial, como base para la conservación del patrimonio histórico, cultural y social, que es imprescindible legar a las futuras generaciones.

\section{REFERENCIAS BIBLIOGRÁFICAS}

ANTEQUERA, Antonio. La situación del patrimonio urbano en la periferia de la gran ciudad: patrimonio oficial versus patrimonio identitario. El caso de Carabanchel Alto (Madrid). Escuela Técnica Superior de Arquitectura de Madrid - Universidad Politécnica de Madrid, 2015.

CABALLERO Rivacoba, María Teresa y YORDI García, Mirtha. Desarrollo social y trabajo comunitario. Teoríametodología y práctica cubana. Camagüey: Editorial Ácana, 2009.

CHAOS, Mabel. La identidad cultural en el centro de la gestión turística de los núcleos monumentales. En: GÓMEZ, Lourdes y NIGLIO, Olimpia, Conservación de centros históricos en Cuba. Volumen II. Roma: Editorial edAEsempi di Architettura, Aracne Editrice, 2015, pp. 663- 676.

ESQUIVEL, Diango. Lineamientos de conservación de los edificios multifamiliares del movimiento moderno del centro histórico de la ciudad de Camagüey. Tesis Máster en Conservación de Centros Históricos y Rehabilitación del Patrimonio Edificado. Facultad de Arquitectura de Camagüey, 2011.

GUERRA, Marisela, MORALES, Carlos Manuel., DE LA CRUZ, Suzel. Educar para la conservación de la biodiversidad en los parques urbanos. Transformación, enerojunio 2014. , vol. 1, n 10 pág. 29 - 39.

MANCEBO, Ingrid., CHAOS, Mabel Teresa y MATAMOROS, Mabel. Impacto de las transformaciones en el hábitat de ciudades patrimoniales. Memorias del X Simposio Internacional Desafíos en el Manejo y Gestión de las Ciudades. Camagüey, febrero del 2016, pág.1- 2.

RODRÍGUEZ, Eduardo Luis. La década incógnita. Los cincuenta: modernidad, identidad, y algo más. Arquitectura Cuba, 1997, n³76, pp. 36-43.

RIGOL, Isabel y ROJAS, Ángela. Conservación patrimonial: teoría y crítica, Cuba: Universidad de La Habana, 2015.

SALGADO, Antonio. La Vivienda. En Busca del Sentido Perdido. En: Narváez, Adolfo. Hábitat y vivienda en América. Ciudad Universitaria, México: Universidad Autónoma de Nuevo León, 2002, pp. 22-24.

SEGRE, Roberto. Lectura crítica del entorno cubano. La Habana: Editorial Letras Cubanas,1990. 